\title{
Theoretical and experimental study of metastable solid solutions and phase stability within the immiscible Ag-Mo binary system
}

Kostas Sarakinos, Grzegorz Greczynski, Viktor Elofsson, Daniel Magnfält, Hans Högberg and Björn Alling

\section{Linköping University Post Print}

\section{Tweet}

N.B.: When citing this work, cite the original article.

Original Publication:

Kostas Sarakinos, Grzegorz Greczynski, Viktor Elofsson, Daniel Magnfält, Hans Högberg and Björn Alling, Theoretical and experimental study of metastable solid solutions and phase stability within the immiscible Ag-Mo binary system, 2016, Journal of Applied Physics, (119), 9, 095303.

http://dx.doi.org/10.1063/1.4942840

Copyright: American Institute of Physics (AIP)

http://www.aip.org/

Postprint available at: Linköping University Electronic Press

http://urn.kb.se/resolve?urn=urn:nbn:se:liu:diva-127275 
Theoretical and experimental study of metastable solid solutions and phase stability within the immiscible Ag-Mo binary system

K. Sarakinos ${ }^{1, *}$, G. Greczynski², V. Elofsson ${ }^{1}$, D. Magnfält ${ }^{1}$, H. Högberg ${ }^{2}$, and B. Alling ${ }^{2,3}$

${ }^{1}$ Nanoscale Engineering Division, Department of Physics, Chemistry, and Biology (IFM), Linköping University, SE-58183, Linköping, Sweden.

${ }^{2}$ Thin Film Physics Division, Department of Physics, Chemistry, and Biology (IFM), Linköping University, SE-58183, Linköping, Sweden.

${ }^{3}$ Max-Planck-Institut für Eisenforschung GmbH, D-40237 Düsseldorf, Germany.

*Corresponding author (e-mail: kostas.sarakinos@.liu.se)

\section{Abstract}

Metastable solid solutions are phases that are synthesized far from thermodynamic equilibrium and offer a versatile route to design materials with tailor-made functionalities. One of the most investigated classes of metastable solid solutions with widespread technological implications is vapor deposited ternary transition metal ceramic thin films (i.e., nitrides, carbides and borides). The vapor-based synthesis of these ceramic phases involves complex and difficult to control chemical interactions of the vapor species with the growing film surface, which often makes the fundamental understanding of the composition-properties relations a challenging task. Hence, in the present study, we investigate the phase stability within an immiscible binary thin film system that offers a simpler synthesis chemistry, i.e. the Ag-Mo system. We employ magnetron co-sputtering to grow $\mathrm{Ag}_{1-\mathrm{x}} \mathrm{Mox}$ thin films over the entire composition range 
along with $x$-ray probes to investigate the films structure and bonding properties. Concurrently, we use density functional theory calculations to predict phase stability and determine the effect of chemical composition on the lattice volume and the electronic properties of Ag-Mo solid solutions. Our combined theoretical and experimental data show that Mo-rich films $(x \geq \sim 0.54)$ form bcc Mo-Ag metastable solid solutions. Furthermore, for Ag-rich compositions ( $x \leq \sim 0.21$ ), our data can be interpreted as Mo not being dissolved in the Ag fcc lattice. All in all, our data show an asymmetry with regards to the mutual solubility of $\mathrm{Ag}$ and $\mathrm{Mo}$ in the two crystal structures, i.e., $\mathrm{Ag}$ has a larger propensity for dissolving in the bcc-Mo lattice as compared to Mo in the fcc-Ag lattice. We explain these findings in light of isostructural short-range clustering that induces energy difference between the two (fcc and bcc) metastable phases. We also suggest, that the phase stability can be explained by the larger atomic mobility of Ag atoms as compared to that of Mo. The mechanisms suggested herein may be of relevance for explaining phase stability data in a number of metastable alloys, such as ternary transition metal-aluminum-nitride systems.

\section{INTRODUCTION}

Historically, alloying has been a key route to develop materials with tailor-made properties within physical metallurgy, semi-conductor physics, and ceramic materials. The alloys and compounds that are accessible to equilibrium synthesis are mapped out in the phase diagram literature currently covering almost all binary systems and a continuously growing number of multinary systems. However, contemporary materials science and technology also rely to a large extent on non-equilibrium synthesis routes in which quenching, ${ }^{1}$ use of large external pressures, ${ }^{2}$ or atom-by-atom growth of thin films from the vapor phase ${ }^{3}$ impose limited atomic assembly kinetics that enables the 
formation of metastable phases against the prediction of equilibrium thermodynamics. A characteristic example with widespread scientific and technological implications is the possibility to use physical vapor deposition techniques to synthesize metastable solid solutions in ternary metal nitride,,$^{4,5,6}$ carbide $^{7}$ and boride ${ }^{8}$ systems, in which thermodynamics dictates the existence of miscibility gap over a large composition range up to the melting point. In these solid solutions, substitution of host metal lattice atoms by a second element induces local variations in atomic size and electronic configuration, which along with the configuration of the substitutional atoms in the host lattice affects strain at nanometer length scale, charge distribution and electronic density of states. These phenomena are leveraged to design new materials with tailormade phase stability, ${ }^{9}$ decomposition pathways, ${ }^{10}$ optical,,${ }^{11}$ magnetic,${ }^{12}$ and mechanical ${ }^{13}$ properties of the solid solution.

The possibility to synthesize metastable solid solutions in a knowledge-based manner is imperative to extend the reach of materials design for property tuning. This requires the development of a fundamental understanding of how the size, electronic configuration and spatial distribution of the constituent atoms affect stability and physical attributes of these phases. This is a rather challenging task for ternary ceramic systems, i.e., nitrides, carbides and borides, in which the chemistry in the vapor phase and on the growing surface also plays an important role for structure formation and physical properties of these materials. For instance, in the case of metal nitrides, the chemical nature of nitrogen species used for their vapor-based synthesis (i.e., whether nitrogen is in atomic or molecular form) affects diffusion pathways of adatoms and admolecules ${ }^{14,15}$ and hence the crystallographic texture ${ }^{16}$ and the configurational disorder in the metal sublattice..$^{17,18}$ It is therefore evident that a fundamental understanding of 
the composition-property relations in metastable solid solutions may be greatly facilitated by the use of material systems and synthesis methods characterized by simpler and easier to control surface and gas phase chemistry. These requirements are fulfilled by binary alloys consisting of noble metals $(\mathrm{Cu}, \mathrm{Ag}$, and $\mathrm{Au})$ and 5 - and 6group transition metals $(\mathrm{V}, \mathrm{Nb}, \mathrm{Ta}, \mathrm{Cr}, \mathrm{Mo}$, and $\mathrm{W})$; these alloys exhibit a positive mixing enthalpy (i.e., they are practically immiscible in equilibrium over the entire composition range $)^{19}$, while navigation between and across the transition metal groups allows for varying independently and systematically atomic sizes and valence electron number. Furthermore, synthesis of noble-transition metal compounds using nonequilibrium vapor phase condensation processes (e.g., magnetron sputtering) offers the advantage of controlled and simple (no presence of reactive species is required) growth chemistry, ability to in a flexible manner scan the chemical composition space and tune the atomic assembly kinetics. This synthesis concept combined with first principle calculations, offers a powerful research strategy for gaining insight on phase stability and physical properties of metastable solid solutions. The viability of this strategy is explored in the present study, by investigating the phase formation and stability of binary $\mathrm{Ag}-\mathrm{Mo}$ thin films synthesized from vapor phase by means of magnetron co-sputtering. We employ first principle calculations to predict the phase stability of the Ag-Mo system and determine the effect of composition on the lattice volume and electronic properties of metastable fcc- and bcc-Ag1-xMox $(0 \leq x \leq 1)$ phases. Furthermore, we use x-ray probes to experimentally study the phase formation and bonding properties of the Ag-Mo films. Combining experimental and theoretical data we show the formation of metastable bcc-Ag1-xMox for $x \geq \sim 0.54$, while for $x \leq \sim 0.21$ our data seemingly support that Mo is not dissolved in the fcc-Ag lattice. We suggest that the stronger tendency for phase separation observed in Ag-rich films may be explained 
by: (i) energetic differences associated with short-range isostructural clustering of $\mathrm{Ag}$ and Mo atoms and (ii) higher mobility of $\mathrm{Ag}$ atoms as compared to that of Mo atoms during film growth.

\section{RESEARCH STRATEGY}

\section{A. FIRST PRINCIPLE CALCULATIONS}

First-principle calculations within the framework of density functional theory (DFT) were performed in order to shed light to the relative stabilities of fcc and bcc Ag1-xMox disordered solid solutions and calculate their lattice spacing, and energy shifts of their core-level electronic states. The latter two produce a fingerprint of solid solution formation and facilitate comparison with experiments in which it is not known a priori if synthesis results in solid solution or phase separated thin film samples. The calculations were performed using the Projector Augmented-wave (PAW) method $^{20}$ as implemented in the Vienna Ab-Initio Simulation package (VASP) ${ }^{21,22}$. The generalized gradient approximation (GGA) ${ }^{23}$ was used for exchange-correlation effects. The alloys were modelled using the special quasi-random structure ${ }^{24}$ method with 64-atom supercells created to mimic the pair correlation functions of an ideal random alloy on the first 8 coordination shells. In our supercells we used a $5 \times 5 \times 5$ mesh of k-point distributed with the Monkhorst-Pack scheme, while the plane-wave cutoff was $400 \mathrm{eV}$ in all cases. Bcc and fcc Ag1-xMox supercells with the compositions $x=0.125,0.25$, $0.375,0.50,0.625,0.75,0.875$ were used together with pure $\mathrm{Ag}$ and Mo, and internal atomic positions were fully relaxed. Mixing enthalpies are calculated according to the expression

$$
\Delta H(x)=E\left(A g_{1-x} M o_{x}\right)-x E(b c c-M o)-(1-x) E(f c c-A g),
$$


where $E\left(A g_{1-x} M o_{x}\right)$ is the energy of the metastable solid solution (this being either bcc or fcc) and $E(b c c-M o) / E(f c c-A g)$ is the energy of the equilibrium bcc-Mo/fcc-Ag phases all taken at the equilibrium volume of each particular concentration. Simulations of the core-level energy shifts of $\mathrm{Ag}$ and Mo were performed within the $\mathrm{Z}+1$ approximation. ${ }^{25}$ In this method core-electron excitation process and the effect of a core-level hole screened by an extra valence electron is approximated with an extra proton and an extra electron. Thus, for each Ag atom in the supercells, a calculation is made with a $\mathrm{Cd}$ atom replacing it, and for each Mo atom, a calculation is done with a Tc atom replacing it. The energy change for each $Z+1$ configuration of a particular alloy concentration and each crystal structure is then averaged and the difference to the $Z+1$ change in energy of the pure elements is identified as the simulated core level shift. This methodology has previously been used e.g., by Marten et al..$^{25}$ to calculate core-level shifts in Cu-Pd and Ag-Pd alloys. In order to assess the statistical significance in the trends of the DFT core-level shifts as function of the composition, $95 \%$ confidence intervals were calculated for each mean value based on the spread in shifts of atoms in different local environments.

\section{B. FILM SYNTHESIS}

Ag-Mo compound films were synthesized from the vapor phase using dc magnetron co-sputtering. Depositions were performed in an ultra-high vacuum stainless steel chamber with a base pressure of the order of $10^{-7} \mathrm{~Pa}$ to minimize the impurity incorporation in the films. For the experiments, two magnetrons equipped with $\mathrm{Ag}$ and Mo targets (99.99\% purity for both metals) with a diameter of $75 \mathrm{~mm}$ and respective thicknesses of 6 and $3 \mathrm{~mm}$ were used. The Ag target-to-substrate distance was 125 $\mathrm{mm}$, while the corresponding value for the Mo target was $75 \mathrm{~mm}$. Both magnetrons 
were positioned at an angle of $40^{\circ}$ with respect to the substrate normal. The magnetrons were fed by separate dc generators operating at constant power mode. Plasma was ignited using $\mathrm{Ar}$ as a sputtering gas at a working pressure of $0.67 \mathrm{~Pa}$. Films were grown on $\mathrm{Si}(100)$ substrates covered by a thermally grown $\mathrm{SiO}_{2}$ layer with a thickness of $300 \mathrm{~nm}$. The substrates were electrically floating and no intentional heating was used during deposition. However, based on previous data concerning growth of $\mathrm{Ag}$ films at conditions similar to those employed in the present study, ${ }^{26}$ we estimate the substrate temperature during deposition to be of the order of $50^{\circ} \mathrm{C}$. The composition of $\mathrm{Ag}_{1-\mathrm{x} M 0 x}$ films was varied from $\mathrm{x}=0$ (pure $\mathrm{Ag}$ ) to $\mathrm{x}=1$ (pure Mo) by independently adjusting the power values applied on the Ag and Mo cathodes using deposition rate data (determined by means of $\mathrm{x}$-ray reflectometry as detailed in section II.C) for monolithic films grown for various powers applied on $\mathrm{Ag}$ and Mo cathode (maximum values 20 and $60 \mathrm{~W}$ respectively). The targeted elemental compositions were confirmed by x-ray photoelectron spectroscopy (XPS) measurements (see section II.C). All Ag1-xMox films were grown at a constant deposition rate of $\sim 0.1 \mathrm{nms}^{-1}$ and deposition times were adjusted to achieve a thickness of $\sim 120 \mathrm{~nm}$.

\section{FILM CHARACTERIZATION}

The crystal structure and phase composition of the $\mathrm{Ag}_{1-\mathrm{x}} \mathrm{Mox}_{\mathrm{x}}$ films were determined by means of X-ray diffractometry (XRD). XRD measurements in the Bragg-Brentano geometry (angular $2 \theta$ range 30 to $50^{\circ}$ ) were performed in a four-circle diffractometer (Panalytical) using CuKa-radiation (1.5406 ̊). Lorentz functions were fitted to the recorded diffraction peaks to determine peak position and thus lattice parameter of the films. X-ray reflectrometry (XRR) measurements were performed in the same 
apparatus and the XRR data were analyzed using the X'pert Reflectivity software (Panalytical) to determine the film thickness.

The bonding properties and compositions of the films were investigated by means of x-ray photoelectron spectroscopy (XPS). XPS measurements were performed in an Axis Ultra DLD spectrometer from Kratos Analytical, with a base pressure of $1.5 \times 10^{-7}$ $\mathrm{Pa}$, using monochromatic Al Ka radiation ( $\mathrm{hv}=1486.6 \mathrm{eV})$. The binding energy scale was previously calibrated by setting the position of the Fermi edge of a sputter-cleaned Ag sample to $0 \mathrm{eV}^{27}$ resulting in the position of the $\mathrm{Ag} 3 \mathrm{~d}_{5 / 2}$ core-level peak at 368.3 eV. ${ }^{28}$ Prior to analyses, samples were sputter-cleaned with $4 \mathrm{keV} \mathrm{Ar}^{+}$ions incident at an angle of $70^{\circ}$ with respect to the surface normal. Monte-Carlo simulations of the interactions of $\mathrm{Ar}^{+}$ions with $\mathrm{Ag}$ and Mo atomic assemblies (using the SRIM code) ${ }^{29}$ at the conditions used for sputter cleaning, showed that the mean $\mathrm{Ag}$ and Mo projected recoil depth is of the order of $25 \AA$. Concurrently, the probing depth of samples for $\mathrm{Ag}$ $3 d$ and Mo $3 d$ core levels is $\sim 50 \AA^{30}$, i.e., a significant fraction of the probed volume remains intact after sputter cleaning and it is hence representative of the original sample composition. Ag 3d and Mo 3d core-level (narrow energy range) spectra were acquired with a pass energy $E_{\text {pass }}=20 \mathrm{eV}$, while for survey (wide energy range) scans, $E_{\text {pass }}=160 \mathrm{eV}$ was used. Quantification was performed using Casa XPS software (version 2.3.16), based upon peak areas from narrow energy range scans which are corrected for (i) the energy-dependent transmission function of the spectrometer, and (ii) the effect of kinetic-energy-dependent electron mean free paths. Elemental sensitivity factors are supplied by Kratos Analytical Ltd. ${ }^{31}$ 


\section{RESULTS AND DISCUSSION}

Figure 1 shows the calculated mixing enthalpies, at zero pressure, of bcc and fcc

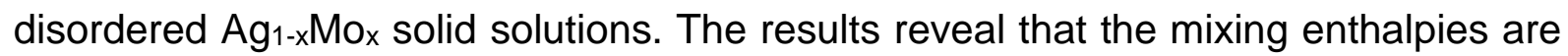
positive for all compositions and for both crystal structures. The fcc alloys have a lower mixing enthalpy than the bcc alloys for approximately $x<0.47$ while at higher $x$ bcc alloys have lower mixing enthalpy. Due to the large positive values of the mixing enthalpies, the configurational entropy term of the Gibbs free energy $(G=H-$ $\left.T S_{\text {config }}\right)^{32}$ would only contribute to an equilibrium inter-solubility less than $1 \%$ in each respective phase within the temperature range $T=300-700 \mathrm{~K}$ being relevant for thin film growth. This finding is in line with the negligible equilibrium solubility according to experimentally-determined phase diagram literature ${ }^{19}$ and confirms the suitability of Ag1-xMox alloys as metastable model system for our studies. Furthermore, our calculations show that there is a thermodynamic driving force for decomposition of (i) Ag-rich fcc alloys into isostructural Ag-rich and Mo-rich domains (fcc $\left.\rightarrow \mathrm{fcc}^{\prime}+\mathrm{fcc}^{\prime \prime}\right)$ and (ii) Mo-rich bcc alloys into isostructural Mo-rich and Ag-rich domains (bcc $\rightarrow$ bcc $^{\prime}+$ bcc") .

Representative XRD patterns of as-deposited $\mathrm{Ag}_{1-\mathrm{x} \text { Mox }}$ thin films for various $\mathrm{x}$ values ( $x$ determined by XPS) are shown in Fig. 2 . There it can be seen that by increasing the Mo context $x$ in the films, the diffraction peaks shift from the angular position of the fcc$\mathrm{Ag}(111)^{33}$ intensity maximum to that of the bcc-Mo (110) ${ }^{34}$ (both indicated by the vertical dashed lines in Fig. 2). In addition Fig. 2 reveals that XRD patterns corresponding to either pure films (Figs. 2 (a) and (i)) or films in which Ag/Mo are the majority species (Figs. 2 (b) and (g)) exhibit rather sharp diffraction peaks. This is not the case in XRD patterns from binary films with comparable fractions of $\mathrm{Ag}$ and Mo 
$(x=0.4,0.45,0.49$ and 0.54 in Figs. $2(c),(d),(e)$ and (f), respectively) which exhibit considerably broader diffraction peaks. It is also seen that the diffraction peaks corresponding to $\mathrm{x}=0.4$ and 0.45 (Figs. 2 (c) and (d), respectively) are asymmetric and can only be fitted with two Lorentzian functions. The first function is centered at angles close to that of the $\mathrm{Ag}$ (111) intensity maximum position, while the second one exhibits a maximum at higher angles. On the contrary, peaks from films with $x=0.21,0.49,0.54$ and 0.83 (Figs. 2 (b), (e), (f) and (g), respectively) are symmetric and can be sufficiently described with one Lorentz function. Hence, based on the evolution of the position and shape of the XRD peaks as function of film composition, we conclude that the diffraction peaks in the range $x \leq \sim 0.21$ stem from the fcc- $A g$ (111) reflection, while for $x>\sim 0.45$ the diffraction peaks seemingly stem from the bcc-Mo (110) reflection. Moreover, for the transition range $\sim 0.21<x \leq \sim 0.45$ the low and high angle component of the recorded diffraction peak originate from the fcc-Ag (111) and bcc-Mo (110) reflections, respectively.

To better understand the effect of the Mo content $x$ on the binary alloy phase composition, XRD data were analyzed by calculating the lattice parameters $a,(\mathrm{a}=$ $d_{h k l} /\left(\sqrt{h^{2}+k^{2}+l^{2}}\right)$ ), of the crystalline phases that contribute to the recorded diffraction patterns. In light of the findings in Fig. 2 and the discussion in the previous paragraph, the interplanar spacing, $d_{h l k}$, for films with compositions $x \leq \sim 0.21$ and x> 0.45 were calculated using $\mathrm{Ag}$ (111) and Mo (110) reflections, respectively. For XRD patterns corresponding to films with compositions in the range $\sim 0.21<\mathrm{X} \leq \sim 0.45$, two lattice constants were calculated using the $\mathrm{Ag}(111)$ and the Mo (110) plane for the low- and high-angle component of the diffraction peak, respectively. The results 
are plotted in Fig. 3 along with the corresponding lattice constant values calculated by DFT assuming perfect solid solution of Mo into fcc-Ag lattice and Ag into bcc-Mo lattice. There it is observed that the lattice parameters calculated by DFT are systematically overestimated with regards to their experimentally determined counterparts. This is a well-known effect when using the Generalized Gradient Approximation, which however does not impair our ability to compare measured and calculated lattice parameters with regards to their trends as function of composition, as detailed in the following. Figure 3 shows that for $x \leq \sim 0.21$ the lattice parameter, a, calculated using the fcc-Ag (111) planes is $\sim 4.085 \AA$-which is nearly identical to the reference fcc Ag value (4.086 $\AA)^{33}$ —and gradually decreases down to $\sim 4.035 \AA$ when $\mathrm{x}$ increases further from $\sim 0.21$ to $\sim 0.45$. Concurrently, the lattice parameter calculated using the bcc-Mo (111) planes exhibits a continuous decrease from $\sim 3.251 \AA$ down to $\sim 3.147 \AA$ (identical to the bcc Mo reference value ${ }^{34}$ ) when $\mathrm{x}$ is increased from $\sim 0.39$ to 1 . The experimentally determined trends with respect to lattice constant are corroborated by DFT data in the $x$ range $\sim 0.21$ to 1 . For $x \leq \sim 0.21$, theory and experiments are not consistent, since DFT shows a decrease in the fcc lattice volume upon Mo incorporation. The latter trend, as well as the predicted increase of the bcc lattice size when Mo is substituted by Ag, can be explained by the atomic radius of Ag being larger than that of Mo (1.44 and $1.39 \AA$, respectively for coordination number 12$)^{35}$. Hence, the comparison of the qualitative behavior of theoretical and experimental data in Fig. 3 provide evidence for the formation of metastable bcc-Ag1-xMox alloys for $x \geq \sim 0.54$. Moreover, a closer inspection of the diffraction pattern presented in Fig. 2 (f) $(x=0.54)$ reveals a weak peak at the position of fcc-Ag (111) reflection. This finding suggests that excess $\mathrm{Ag}$ atoms that cannot be dissolved in the bcc-Mo lattice may segregate forming pure Ag domains. A further decrease of the $x$ value down to 0.49 (Fig. 2 (e)) causes the observed diffraction 
peak to become significantly broader as compared with the diffraction peaks in Figs. 2 (f), (g) and (i). This broadening may be explained by an overlap of the bcc-Ag1-xMox peak with another peak at lower angular position emanating from (i) Ag segregating as pure fcc phase as in the case of Fig. 2 (f) and/or (ii) metastable fcc-Ag ${ }_{1-x M 0_{x}}$ solid solution as in the range $\sim 0.21<x \leq \sim 0.45$ where $X R D$ data indicate (see Figs. 2 (c) and (d)) that both metastable fcc and bcc phases coexist. Since the data in Fig. 2 (e) do not provide unambiguous evidence in favor of only one of the scenarios (i) and (ii) we argue that the diffraction pattern in Fig. $2(\mathrm{e})(\mathrm{x}=0.49)$ corresponds to the transition zone between the bcc-Ag${ }_{1-x} \mathrm{Mox}_{\mathrm{x}} \quad(\mathrm{x} \geq \sim 0.54)$ and fcc-Ag $1-\mathrm{x} \mathrm{Mox}_{\mathrm{x}} / \mathrm{bcc}-\mathrm{Ag}_{1-\mathrm{x} M o x}$ $(\sim 0.21<x \leq \sim 0.45)$ phase composition regimes.

In general, the angular position of peaks in XRD patterns of multinary thin films is determined not only by the film composition but also by the stress state of the film. Mo films grown at conditions very similar to those employed in the present study, have been found to be under biaxial tensile stress while exhibiting marginal expansion of the lattice in the out-of-plane direction. ${ }^{36}$ This is because at conditions of limited atomic mobility, e.g., at close-to-room-temperature Mo deposition, underdense grain boundaries form which cause attraction between neighboring grains and thereby result in tensile stress generation. ${ }^{37}$ Concurrently, $\mathrm{Ar}^{+}$ions are backscattered from the Mo target and impinge on the surface of the growing film as energetic neutrals creating point defects and a hydrostatic stress contribution; the latter counteract the effect of the tensile stresses leading to a small lattice dilation along the growth direction. ${ }^{36,38}$ In contrast to Mo, growth of $\mathrm{Ag}$ near room temperature results in high atomic mobility. At these conditions, compressive stress builds up during growth as adatoms diffuse into the grain boundaries driven by a chemical potential difference between the surface 
that is supersaturated with adatoms and the grain boundary. This growth stress is relaxed upon growth interruption due to outdiffusion of atoms from the grain boundaries while grain growth, which leads to tensile stress, takes place leading to essentially stress free films. ${ }^{39,40,41}$ Backscattered $\mathrm{Ar}^{+}$ions may also contribute to compressive stress generation in Ag films. Given the similar masses of $\mathrm{Ag}$ and Mo, the energy and flux of backscattered $\operatorname{Ar}$ species is expected to be similar resulting in a similar hydrostatic stress contribution for all films. Hence, based on the arguments presented above, we conclude that the effect of intrinsic stresses on the out-of-plane lattice parameter is not the main contributor to the trends observed in Fig. 3. Thus, the lack of consistency observed between theory and experiment for $x \leq \sim 0.21$ in Fig. 3 can be interpreted as Mo not being dissolved in the fcc-Ag lattice. In this case, a possible explanation is that a pure fcc-Ag phase coexists with an x-ray amorphous Mo-based phase. The latter may also explain the lack of a Mo-bcc signature in the corresponding diffraction patterns in Fig. 2. However, it should be noted that the DFT calculated change of the lattice parameter for the case of metastable fcc-Ag ${ }_{1-\mathrm{x}} \mathrm{Mox}$ solid solution in the range $0<x \leq \sim 0.21$ is much smaller as compared to the change for metastable

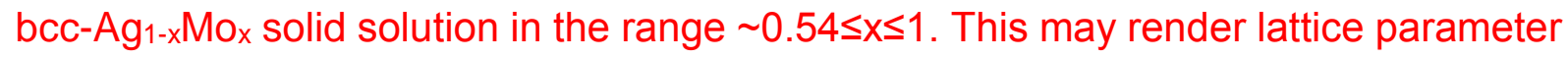
changes in Ag-rich films difficult to detect by XRD, i.e., formation of metastable fcc$\mathrm{Ag}_{1-\mathrm{x}} \mathrm{Mox}$ solid solution cannot be ruled out unambiguously. Thus future studies focused on the Ag-rich compositions are beneficial for shedding additional light to the structure formation of this binary system. In any case and irrespective of whether only one or both scenarios outlined above explain our data in the composition range $x$ $\leq \sim 0.21$, our findings clearly show an asymmetry with regards to solubility, i.e., Ag has a larger propensity for dissolving in the bcc-Mo lattice as compared to Mo in the fccAg lattice. 
XPS was used to correlate structural data to changes in the local chemical environment. For this purpose the effect of chemical composition on the $\mathrm{Ag} 3 \mathrm{~d}$ and the Mo 3d core level binding energy (BE) was investigated. The data revealed no discernible effect of the composition on the BE and shape of the $\mathrm{Ag} 3 \mathrm{~d}$ peak present at $368.3 \mathrm{eV}$ (see Fig. 4 (a)), which is the reference $\mathrm{Ag} 3 \mathrm{~d}$ peak position. ${ }^{28}$ On the other hand, the Mo $3 d$ core level signal was found to deviate from its reference BE of 228.0 eV once Ag was added to the film (see Fig. 4 (b)). To better visualize this behavior, the binding energy shifts of the $\mathrm{Ag} 3 \mathrm{~d}$ and the Mo $3 \mathrm{~d}$ peaks from their respective reference values are plotted as a function of $x$ in Fig. 4 (c). In the same figure, DFT calculated $3 d$ core level shifts for $\mathrm{Ag}$ and $\mathrm{Mo}$ in bcc-Ag1-xMox and for Mo in fcc-Ag1-xMox are also plotted as a function of $x$. The error bars in the DFT data represent the $95 \%$ confidence intervals calculated by the procedure described in Section II. The error in the experimental XPS data was estimated to be $\pm 0.025 \mathrm{eV}$. In Fig. 4 (c) it is observed that XPS results and DFT calculations are in agreement with respect to the qualitative effect of composition change on the $3 \mathrm{~d}$ core level shifts for both $\mathrm{Ag}$ and Mo. For the case of $\mathrm{Ag}$, the theoretical calculations indicate, that the $\mathrm{Ag} 3 \mathrm{~d} B E$ shifts would be small even if solid solutions were formed, indicating that focus for alloy identification should be on the Mo $3 d$ core level shifts. With regards to Mo, the $3 d$ core level shift value is found to decrease with decreasing $x$ and exhibit tendency for saturation below $x=0.6$, irrespective of local chemical environment (bcc or fcc). This is consistent with the experimentally determined values and, thus, corroborates the conclusion from XRD data that the metastable bcc- and fcc-Ag1-xMox solid solutions coexist in the range $\sim 0.21<x \leq \sim 0.45$. This also implies that Mo $3 d$ core level shift cannot be used to distinguish between the two metastable solid solution phases. Moreover, as previously 
discussed one scenario for explaining the data in the composition range $0<x \leq \sim 0.21$ in Fig. 3, is that Mo is not dissolved in the fcc-Ag lattice since the XRD data presented in Fig. 2 do not show any peaks from bcc-Mo for $x \leq \sim 0.21$. Hence, the saturation observed in the experimental and theoretical $3 d$ core level shifts for $x<0.6$ in Fig. 4 (c) may also indicate that Mo in films with $x \leq \sim 0.21$ forms an amorphous Mo-based phase with local chemical environment similar to that of metastable fcc/bcc-Ag $1-\mathrm{xMox}$.

XPS data have also shown that Mo-rich films (i.e., films with $x>0.8$ ) contain high concentrations of $\mathrm{O}$ and $\mathrm{C}$ contaminants, of the order of 20 and 15 at\% respectively, which gradually decrease to values below 3 at $\%$ for $x<0.8$. High levels of contaminants found in $x>0.8 \mathrm{Ag}_{1-x} \mathrm{Mox}_{\mathrm{x}}$ layers are indicative of an underdense microstructure with high degree of intercolumnar porosity that leads to significant $\mathrm{O}$ and $\mathrm{C}$ incorporation at grain boundaries and upon air exposure. ${ }^{42}$ This is consistent with the notion of limited atomic mobility encountered during the growth of refractory metals close-to-room temperature as previously discussed. $3,36,37$ With increasing Ag content in the films the effective mobility of film forming species increases (as $\mathrm{Ag}$ exhibits higher atomic mobility than Mo at room temperature $)^{37,39,40}$ resulting in a denser microstructure which is less prone to impurity incorporation. The incorporation of $\mathrm{O}$ and $\mathrm{C}$ species in the Morich grains is excluded due to the lack of oxide or carbide signature in the corresponding Mo 3d XPS spectra (not shown here). This also implies that the lattice parameter changes presented in Fig. 3 and the inferred phase composition evolution cannot be attributed to the effect of impurities.

The structure formation in the Ag-Mo system-as well as in other Ag-early transition metal systems which exhibit large positive mixing enthalpy—has been studied in the 
literature in light of synthesis of amorphous alloys by means of ion beam mixing using $\mathrm{Ag} / \mathrm{Mo}$ multilayered thin films as a starting point. ${ }^{43,44} \mathrm{~A}$ combination of molecular dynamics simulations and experimental data ${ }^{45}$ showed that the formation of an amorphous structure is accompanied by metastable fcc and bcc solid solution for $\mathrm{x}$ values below 0.1 and above 0.88 , respectively, in the $\mathrm{Ag}_{1-x \mathrm{Mox}}$ alloy. This is in qualitative agreement with the mixing enthalpy data presented in Fig. 1, which indicate a similar bulk thermodynamic driving force against dissolving Mo into fcc- $\mathrm{Ag}$ and $\mathrm{Ag}$ into bcc-Mo assuming disordered alloys. It is also worth noting in Fig. 1 that pure Mo has a much larger energy difference between the two crystal structures $(0.41 \mathrm{eV} /$ atom $)$ as compared to $\mathrm{Ag}(0.038 \mathrm{eV} /$ atom $)$. This is in agreement with theoretical and experimental studies, where the fcc Mo-phase has been found to be dynamically unstable at low temperatures. ${ }^{46,47,48}$ This means that even though the calculated mixing enthalpies of the Mo-rich bcc and Ag-rich fcc ideal random alloys in Fig. 1 appear symmetric with respect to composition, short-range $\mathrm{Ag}$ and Mo isostructural clustering in the bcc structure could lower the driving force for global phase separation in Mo-rich alloys more than in Ag-rich alloys with fcc structure. This type of clustering is commonly observed in metastable alloys synthesized far from equilibrium ${ }^{49}$, see e.g., the study by Tai et al. ${ }^{50}$ who reported that the high quenching rates during synthesis of Ag-Mo alloys by ion irradiation lead to the formation of $\mathrm{Ag}$-rich and Mo-rich phases that exhibit compositional modulation (i.e., clustering) at length scales of about $100 \mathrm{~nm}$. A similar mechanism, due to the high quenching rates encountered upon the condensation of vapor species on the growing film surface, may also be operative in our case providing a pathway for the formation of bcc Mo-rich solid solutions. 
Apart from thermodynamics, the microstructure of thin films synthesized far-fromequilibrium is also determined by the growth kinetics. In the present case, an accurate and full atomistic description of the kinetic processes that determine atomic arrangement at the nanoscale requires knowledge of the hetero-epitaxial diffusion barriers (e.g., terrace and step-edge diffusion) for the most commonly observed crystallographic surfaces and the compositions of interest within the Ag-Mo system. Neither experimentally nor theoretically determined values for these barriers are reported in the literature, thus in the following we merely present a qualitative description of the kinetic processes that may contribute to the structure evolution of the Ag-Mo films. The two materials are characterized by considerably different diffusion barriers on their most commonly encountered surfaces, i.e., $\sim 0.1^{51}$ and $\sim 0.6 \mathrm{eV}^{52}$ for $\mathrm{Ag}$ and Mo adatoms on $\mathrm{Ag}$ (111) and Mo (110), respectively. This difference can be understood in terms of the bond strength ${ }^{53}$ of the two materials, 1.65 and $4.35 \mathrm{eV}$ for $\mathrm{Ag}$ and Mo respectively which is also reflected upon their melting points ${ }^{54}$ (1235 and $2890 \mathrm{~K}$, respectively). The growth kinetics of Ag-rich alloys is dominated by $\mathrm{Ag}-\mathrm{Ag}$ interactions which leads to a larger effective atomic mobility as opposed to the Mo-rich alloys where surface kinetics of Mo largely determines structure formation and evolution. Thus on the Ag-rich side of the composition space the larger effective atomic mobility may allow for diffusional breakdown of the initially homogeneous structure formed upon vapor condensation ${ }^{54}$ leading to local isostructural clustering and phase segregation. These processes are less facile in the Mo-rich films resulting in a rather stable Mo-bcc lattice in which Ag atoms can dissolve. The picture described above is in agreement with data from Saunders and Miodownik ${ }^{54}$ and Chang et al. ${ }^{55}$ for other immiscible alloy systems, which showed that larger diffusivities and thus longer surface 
diffusion lengths favor the formation of equilibrium phases at the expense of metastable solid solutions.

\section{SUMMARY AND OUTLOOK}

In summary we have grown $\mathrm{Ag}_{1-\mathrm{x}} \mathrm{Mo}_{\mathrm{x}}(0 \leq \mathrm{x} \leq 1)$ thin films over the entire composition range by means of magnetron co-sputtering and studied the film microstructure, lattice size and bonding properties using $\mathrm{x}$-ray probes. Experiments were combined with first principle DFT calculations to predict phase stability and determine the effect of chemical composition on the lattice volume and the core-level energy shifts in metastable fcc and bcc solid solutions. DFT data revealed that the binary Ag-Mo system exhibit large positive values of mixing enthalpy over the entire composition range, confirming the notion from phase diagrams that the two metals exhibit a large miscibility gap. In addition, the theoretical calculations showed that substitution of $\mathrm{Ag}$ by Mo in the fcc lattice results in a decrease of the lattice volume with respect to the equilibrium volume of pure Ag. The opposite effect is observed in the case of bcc Mo. XRD data confirmed the trends predicted by theory for Mo-rich films $(x>\sim 0.45)$ but not for Ag-rich films ( $x \leq \sim 0.21)$. Concurrently, XPS data showed that Mo core energy levels shift in the Mo-rich composition range while Ag core energy levels are constant independent of $x$. These data were found to be consistent with core level shifts calculated from DFT. By combining experimental and theoretical results we have concluded that metastable bcc-Ag1-xMox solid solutions form for $x \geq \sim 0.54$, while our data can be interpreted as Mo not being dissolved in the fcc-Ag lattice for $x \leq \sim 0.21$, i.e., there is an asymmetry with regards to the mutual solubility of $\mathrm{Ag}$ and Mo in the two crystal structures. Moreover, DFT calculations showed that pure Mo has a much larger energy difference between the two crystal structures (fcc and bcc) as compared to Ag. 
This means that even though the calculated mixing enthalpies of the Mo-rich bcc and Ag-rich fcc ideal random alloys appear symmetric with respect to composition, shortrange $\mathrm{Ag}$ and Mo isostructural clustering in the bcc structure could lower the driving force for global phase separation in Mo-rich alloys more than in Ag-rich alloys with fcc structure. This type of isostructural clustering is commonly observed in metastable solid solutions, due to the non-equilibrium character of the processes employed for their synthesis. These processes impose kinetic constraints during film growth which means that Ag-rich films exhibit a higher effective atomic mobility as compared to their Mo-rich counterparts. The latter may also explain the observed difference in phase stability and solubility.

In the course of the previous decades there has been extensive theoretical and experimental studies on the metastable transition metal (TM)-Al-N ternary nitride systems ${ }^{5,6,56,57,58}$ focusing on the maximum solubility of AIN in the rock-salt (TM)N phase, as well as the maximum solubility of (TM)N in the wurtzite phase of AIN. Available data have shown a distinct asymmetry in the metastable solubility between the two crystallographic phases, e.g., in magnetron sputtered $\mathrm{Ti}_{1-\mathrm{x}} \mathrm{A} \mathrm{I}_{\times} \mathrm{N}$, the metastable solubility of $\mathrm{Al}$ in rocksalt-TiN is of the order of $60 \%$ at. Al in the metal sublattice, while the Ti metastable solubility in wurtzite-AIN is very low and rarely reported. ${ }^{59}$ From a theoretical point of view this has been explained by the crossing point of the mixing enthalpy curves ${ }^{56}$ being shifted towards AIN-rich composition at $70 \%$ at. $\mathrm{Al}$ in the metal sublattice. ${ }^{60,61}$ In the case of the $\mathrm{Ag}_{1-x M 0 x}$ binary system investigated in the present study, we also observe a distinct metastable solubility asymmetry, despite the fact that DFT calculations show that the mixing enthalpy curves cross around $50 \%$ at. Mo and are quite symmetric in their respective composition regimes of relative stability. 
Instead, the difference in atomic mobility between Mo and Ag, as well as the higher propensity for the bcc than the fcc alloys to lower their mixing enthalpies by short-range clustering are suggested to be the reason for the solubility asymmetry. These mechanisms may also be relevant for explaining solubility asymmetry in, for example, ternary TM-AI-N systems where anisotropy in mobility of adatoms depending on their chemical nature and film surface termination ${ }^{15,16,17,18}$ may trigger local clustering and change the energetics of the metastable phases as compared to random solid solutions. ${ }^{62}$ Beyond the present study, systematic investigations within the highly immiscible binary alloys consisting of noble metals $(\mathrm{Cu}, \mathrm{Ag}$, and $\mathrm{Au}$ ) with the fcc crystal structure and 5- and 6-group transition metals with the bcc crystal structure (V, Nb, Ta, $\mathrm{Cr}$, Mo, and W) can be used to unravel the effect of atomic size, mobility and electronic configuration on the phase stability mechanisms outlined above.

\section{ACKNOWLEDGEMENTS}

K.S. and V.E. should like to acknowledge Linköping University for financial support through the "LiU Research Fellows Program, 2011-2015" and the "LiU Career Contract (Dnr-LiU-2015-01510), 2015-2020". B.A. acknowledges financial support from the Swedish Research Council (VR) through grant No. 621-2011-4417 and No. 330-20146336. G.G. wishes to acknowledge financial support from the VINN Excellence Center Functional Nanoscale Materials (FunMat). H.H. acknowledges financial support from the Swedish Government Strategic Research Area in Materials Science on Functional Materials at Linköping University (Faculty Grant SFO-Mat-LiU No. 2009-00971). All calculations were performed using supercomputer resources provided by the Swedish National Infrastructure for Computing (SNIC) at the National Supercomputer Centre 
(NSC). The authors wish to thank Prof. Lars Hultman (Linköping University) for his comments on the manuscript.

\section{REFERENCES}

'Y. Q. Cheng and E. Ma, Prog. Mat. Sci. 56, 379 (2011).

${ }^{2} \mathrm{E}$. Boldyrava and D. Przemyslaw (Eds), "High-Pressure Crystallography: From Fundamental Phenomena to Technological Applications", Springer, 2010.

${ }^{3}$ P. Martin (ed), "Handbook of Deposition Tech. for Thin Films and Coatings", Elsevier, 2010.

${ }^{4}$ H. Holleck, Surf. Coat. Tech. 36, 151 (1988).

${ }^{5}$ F. Rovere, D. Music, S. Ershov, M. to Baben, H-G Fuss, P.H. Mayrhofer, and J.M. Schneider, J. Phys. D: Appl. Phys. 43, 035302 (2010).

${ }^{6}$ P.H. Mayrhofer, A. Hörling, L. Karlsson, J. Sjölén, T. Larsson, C. Mitterer, and L. Hultman, Appl. Phys. Lett. 83, 2049 (2003).

7J.-P. Palmquist, J. Birch, and U. Jansson, Thin Solid Films 405, 122 (2002).

${ }^{8}$ H. Euchner and P.H. Mayrhofer, Thin Solid Films 583, 46 (2015).

${ }^{9}$ B. Alling, A. Karimi, I.A. Abrikosov, Surf. Coat. Technol. 203, 883 (2008).

${ }^{10}$ R. Rachbauer, S. Massl, E. Stergar, D. Holec, D. Kiener, J. Keckes, J. Patscheider, M. Stiefel, H. Leitner, and P.H. Mayrhofer, J. Appl. Phys. 110, 013515 (2011).

${ }^{11}$ O. Banakh, M. Balzer, M. Fenker, A. Blatter, Surf. Coat. Technol. 455, 650 (2004).

${ }^{12}$ B. Alling, Phys. Rev. B 82, 054408 (2010).

${ }^{13}$ A. Horling, L. Hultman, M. Oden, J. Sjolen, L. Karlsson, Surf. Coat. Technol. 191, $384(2005)$. 
${ }^{14}$ D. Gall, S. Kodambaka, M.A. Wall, I. Petrov, and J.E. Greene, J. Appl. Phys. 93, $9086(2003)$.

${ }^{15}$ D.G. Sangiovanni, D. Edström, L. Hultman, V. Chirita, I. Petrov, and J.E. Greene, Phys. Rev. B 86, 155443 (2012).

${ }^{16}$ C.-S. Shin, D. Gall, Y.-W. Kim, N. Hellgren, I. Petrov, and J.E. Greene, J. Appl. Phys. 92, 5084 (2002).

${ }^{17}$ B. Alling, P. Steneteg, C. Tholander, F. Tasnádi, I. Petrov, J.E. Greene, and L. Hultman, Phys. Rev. B 85, 245422 (2012).

${ }^{18}$ C. Tholander, B. Alling, F. Tasnádi, J.E. Greene, and L. Hultman, Surf. Sci. 630, 28 (2014).

${ }^{19}$ F. R. de Boer, R. Boom, W. C. M. Mattens, A. R. Miedema, and A. K. Niessen, Cohesion in Metals: Transition Metal Alloy, North-Holland Amsterdam, 1989.

${ }^{20}$ P. E. Blöchl, Phys. Rev. B 50, 17953 (1994).

${ }^{21} \mathrm{G}$. Kresse and J. Furthmüller, Phys. Rev. B 54, 11169 (1996).

${ }^{22}$ G. Kresse and D. Joubert, Phys. Rev. B 59, 1758 (1999).

23J. P. Perdew, K. Burke, and M. Ernzerhof, Phys. Rev. Lett. 77, 3865 (1996).

${ }^{24}$ A. Zunger, S. -H. Wei, L. G. Ferreira, and J. E. Bernard, Phys. Rev. Lett. 65, 353 (1990).

${ }^{25}$ Marten, W. Olovsson, S. I. Simak, and I. A. Abrikosov, Phys. Rev. B 72, 054210 (2005).

${ }^{26}$ B. Lü, V. Elofsson, E.P. Münger, and K. Sarakinos, Appl. Phys. Lett. 105, 163107 (2014)

${ }^{27}$ S. Hüfner, "Photoelectron Spectroscopy: Principles and Applications, $3^{\text {rd }}$ Eds.", Springer-Verlag, Germany, 2010. 
${ }^{28}$ J.F. Moulder, W.F. Stickle, P.E. Sobol, K.D. Bomben, "Handbook of X-ray Photoelectron Spectroscopy", Perkin-Elmer Corporation, Eden Prairie, USA, 1992. ${ }^{29}$ www.srim.org and documentation therein.

${ }^{30}$ S. Tanuma, C. J. Powell and D. R. Penn, Surf. Interf. Anal. 43, 689 (2010).

${ }^{31}$ Kratos Analytical Ltd.: library filename: "casaXPS_KratosAxis-F1s.lib".

${ }^{32} S_{\text {config }}$ can be approximated as $S_{\text {config }}=-k_{B} T[x \ln x-(1-x) \ln (1-x)]$

${ }^{33}$ International Center for Diffraction Data JCPDS, Powder Diffraction File No 00-0040783.

${ }^{34}$ International Center for Diffraction Data JCPDS, Powder Diffraction File No 00-00421120.

35U. Müller, "Inorganic Structural Chemistry, 2nd Edition", Wiley, 2006.

${ }^{36}$ D. Magnfält, G. Abadias, and K. Sarakinos, Appl. Phys. Lett. 103, 051910 (2013).

${ }^{37}$ R. Koch, Surf. Coat. Technol. 204, 1973 (2010).

${ }^{38}$ A. Debelle, G. Abadias, A. Michel, C. Jaouen, and V. Pelosin, J. Vac. Sci. Technol. A 25, 1438 (2007).

${ }^{39}$ G. Abadias, A. Fillon, J.J. Colin, A. Michel, C. Jaouen, Vacuum 100, 26 (2013).

${ }^{40}$ D. Magnfält, V. Elofsson, G. Abadias, U. Helmersson, and K. Sarakinos, J. Phys. D: Appl. Phys. 46, 215303 (2013).

${ }^{41}$ E. Chason, Thin Solid Films 526, 1 (2012).

${ }^{42 G . ~ G r e c z y n s k i, ~ J . ~ L u, ~ I . ~ P e t r o v, ~ J . E . ~ G r e e n e, ~ S . ~ B o l z, ~ W . ~ K o ̈ l k e r, ~ C h . ~ S c h i f f e r s, ~ O . ~}$ Lemmer, and L. Hultman, J. Vac. Sci. Technol. A 32, 041515 (2014).

${ }^{43}$ O. Jin, J. Zhang, and B.X. Liu, Appl. Phys. Lett. 67, 1524 (1995).

${ }^{44}$ O. Jin and B.X. Liu, Nucl. Instr. Method. Phys. Res. B 114, 56 (1996).

${ }^{45}$ K.P. Tai, X.D. Dai., Y.X. Shen, and B.X. Liu, J. Phys. Chem. B 110, 595 (2006). 
${ }^{46}$ A. Dinsdale, CALPHAD: Comput. Coupling Phase Diagrams Thermochem. 15, 317 (1991).

${ }^{47}$ N. Saunders and A. P. Miodowinik, "CALPHAD. Calculations of Phase Diagrams. A Comprehensive Guide", Elsevier Science, Oxford, 1998.

${ }^{48}$ Asker, A. B. Belonoshko, A. S. Mikhaylushkin, and I. A. Abrikosov, Phys. Rev. B 77, 220102(R) (2008).

${ }^{49}$ E. Ma, Prog. Mater. Sci. 50, 413 (2005).

${ }^{50}$ K.P. Tai, X.D. Dai and B.X. Liu, Appl. Phys. Lett. 88, 184103 (2006).

${ }^{51}$ V.R. Dhanak, and D.W. Basset, Surf. Sci. 238, 289 (1990).

${ }^{52 G . ~ A t n c z a k ~ a n d ~ G . ~ E r l i c h, ~ " S u r f a c e ~ D i f f u s i o n . ~ M e t a l s, ~ M e t a l ~ A t o m s, ~ a n d ~ C l u s t e r s ", ~}$ Cambridge University Press, 2010.

53W.M. Haynes (ed.), "CRC Handbook of Chemistry and Physics", CRC Press 2000.

${ }^{54}$ N. Saunders and A.P. Miodownik, J. Mater. Sci. 22, 629 (1987).

${ }^{55}$ K. Chang, M. to Baben, D. Music, D. Lange, H. Bolvardi, and J.M. Schneider, Acta. Mater. 98, 135 (2015).

${ }^{56}$ D. Holec, R. Rachbauer, L. Chen, L. Wang, D. Luef, P.H. Mayrhofer, Surf. Coat. Techno. 206, 1698 (2011).

${ }^{57}$ C. Höglund, J. Birch, B. Alling, J. Bareno, Z. Czigany, P.O.A. Persson, G. Wingqvist, A. Zukauskaite, and L. Hultman, J. Appl. Phys. 107, 123515 (2010).

${ }^{58}$ G. Greczynksi, J. Lu, M.P. Johansson, J. Jensen, I. Petrov, J.E. Greene, and L. Hultman, Surf. Coat. Technol. 206, 4202 (2012).

${ }^{59}$ G. Greczyknsi, J. Lu, J. Jensen, S. Bolz, W. Koelker, Ch. Schiffers, O. Lemmer, J.E. Greene, and L. Hultman, Surf. Coat. Technol., 257, 15 (2014

${ }^{60}$ B. Alling, M. Oden, L. Hultman, and I.A. Abrikosov, Appl. Phys. Lett. 95, 181906 (2009). 
${ }^{61}$ P. H. Mayrhofer, D. Music, and J.M. Schneider, Appl. Phys. Lett. 88, 071922 (2006).

62P.H. Mayrhofer, D. Music, and J.M. Schneider, J. Appl. Phys. 100, 0914906 (2006). 


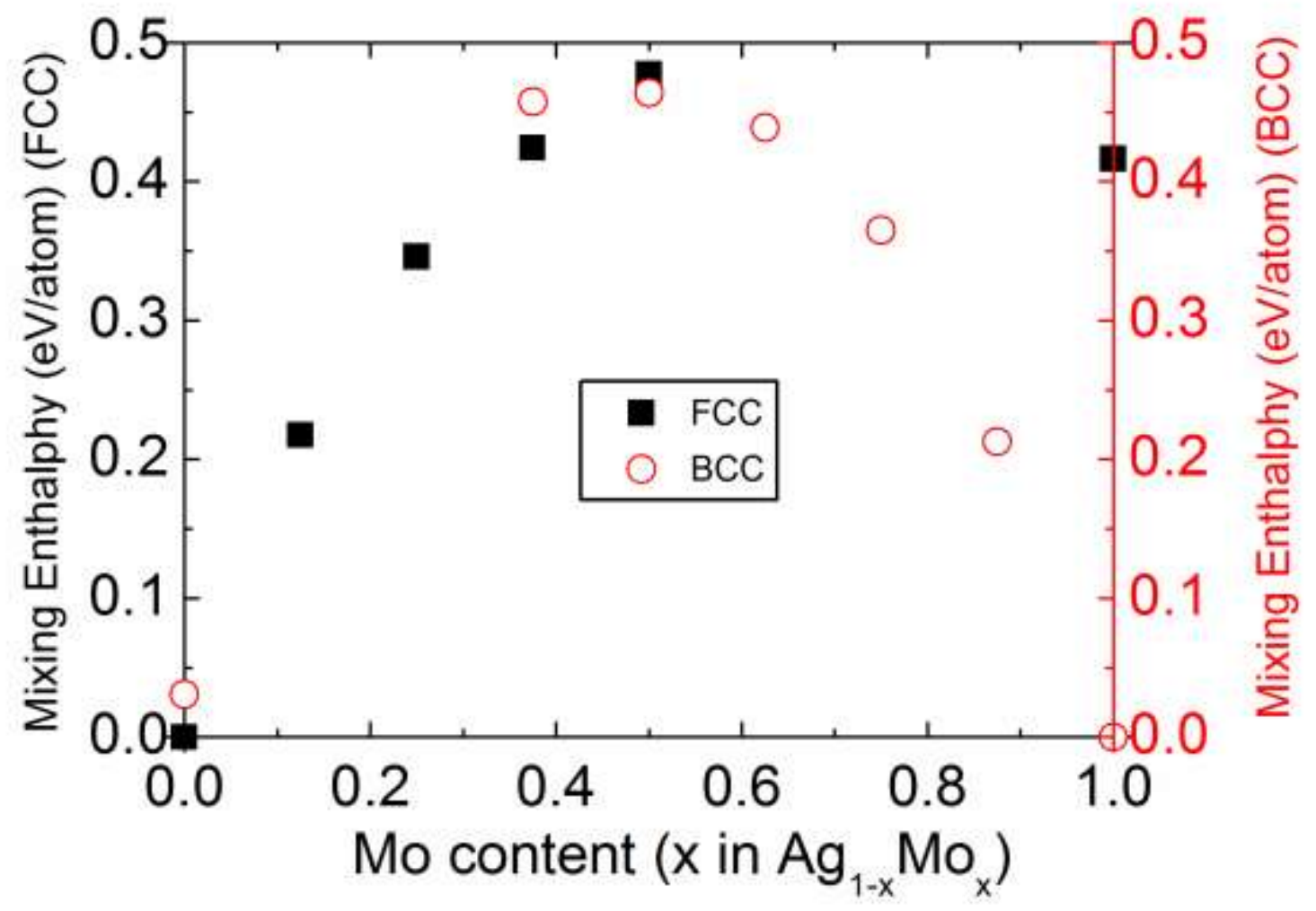

Fig. 1. (Color online) Mixing enthalpy for $\mathrm{Ag}_{1-x M o x} \mathrm{FCC}$ and $\mathrm{BCC}$ alloys calculated using density functional theory. 


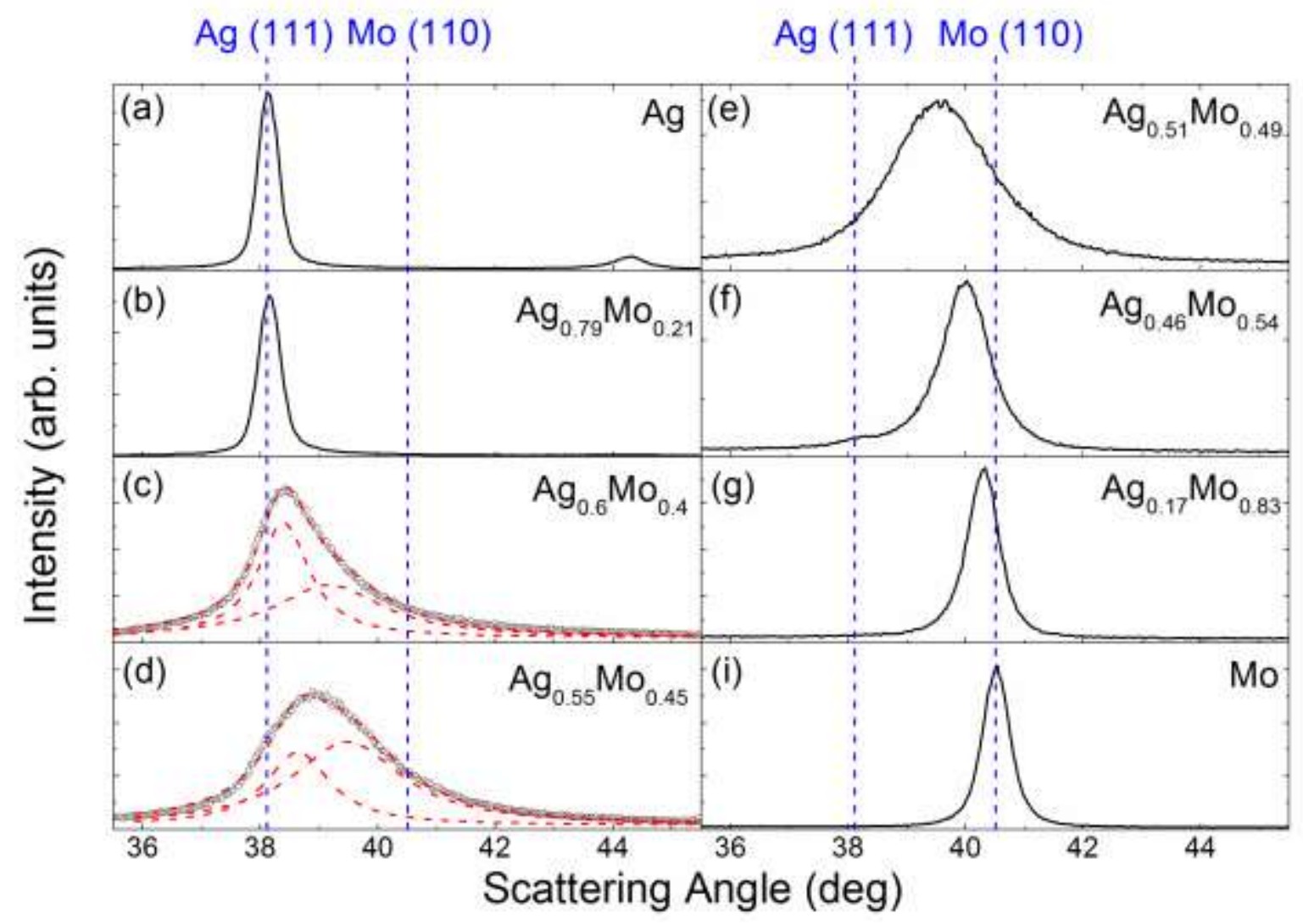

Fig. 2. (Color online) Representative XRD patterns from Ag-rich, Mo-rich and mixed phases. The vertical dashed lines indicate the position of the fcc-Ag (111) and bcc-Mo (110) peak positions. The dashed curves in (c) and (d) represent the Lorentz functions used to fit the XRD peak of the $\mathrm{Ag}_{0.6 \mathrm{Mo}} \mathrm{M}_{0.4}$ and $\mathrm{Ag}_{0.55} \mathrm{M}_{0.45}$ films. 


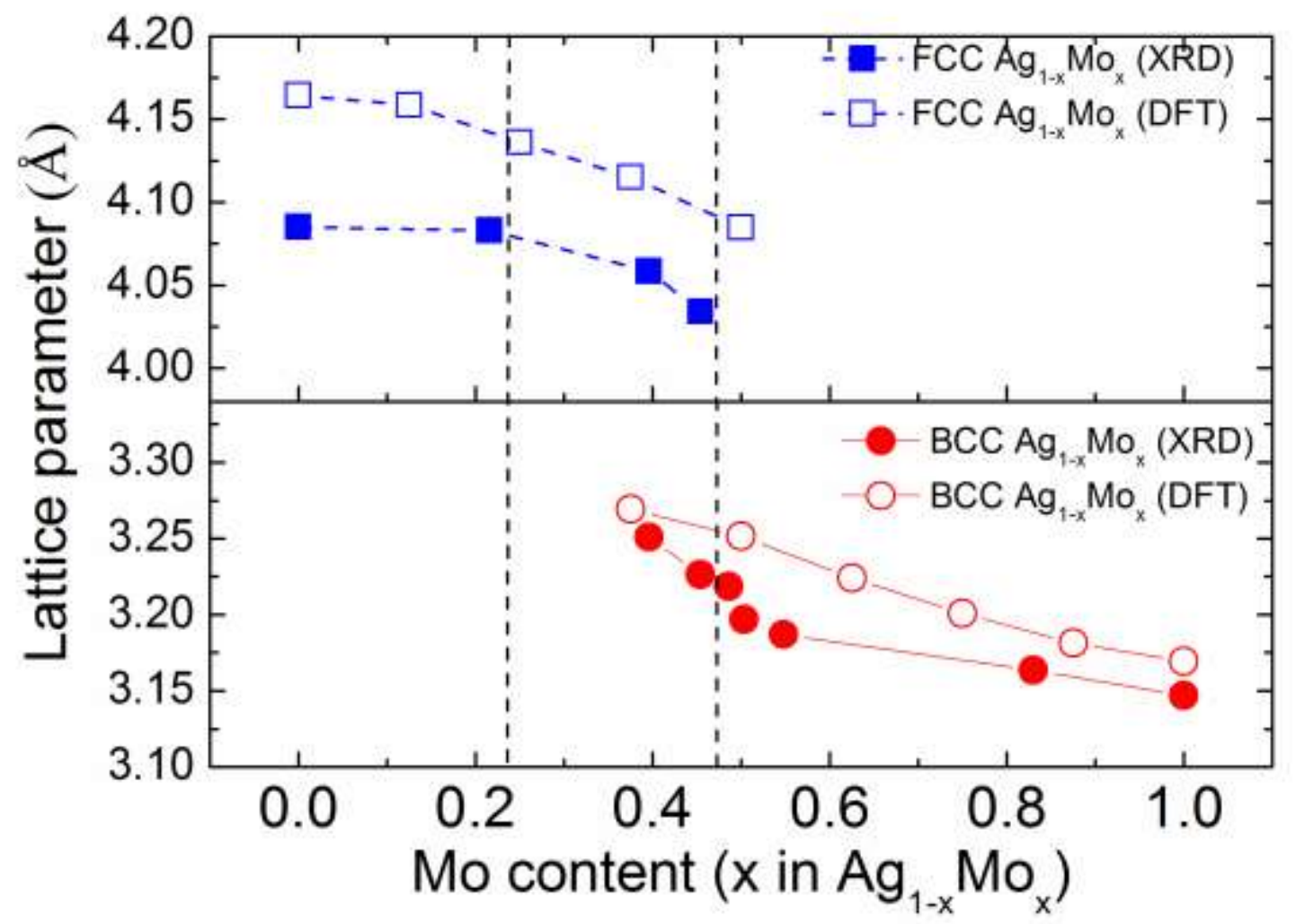

Fig. 3. (Color online) Lattice constants of fcc- and bcc-Ag1-xMox structures (top and bottom panel, respectively). Experimental data (XRD) are represented by filled symbol while data from DFT calculations are represented hollow symbols. The vertical dotted lines define the compositional range (as determined by XRD data) in which the two metastable phases coexist. 

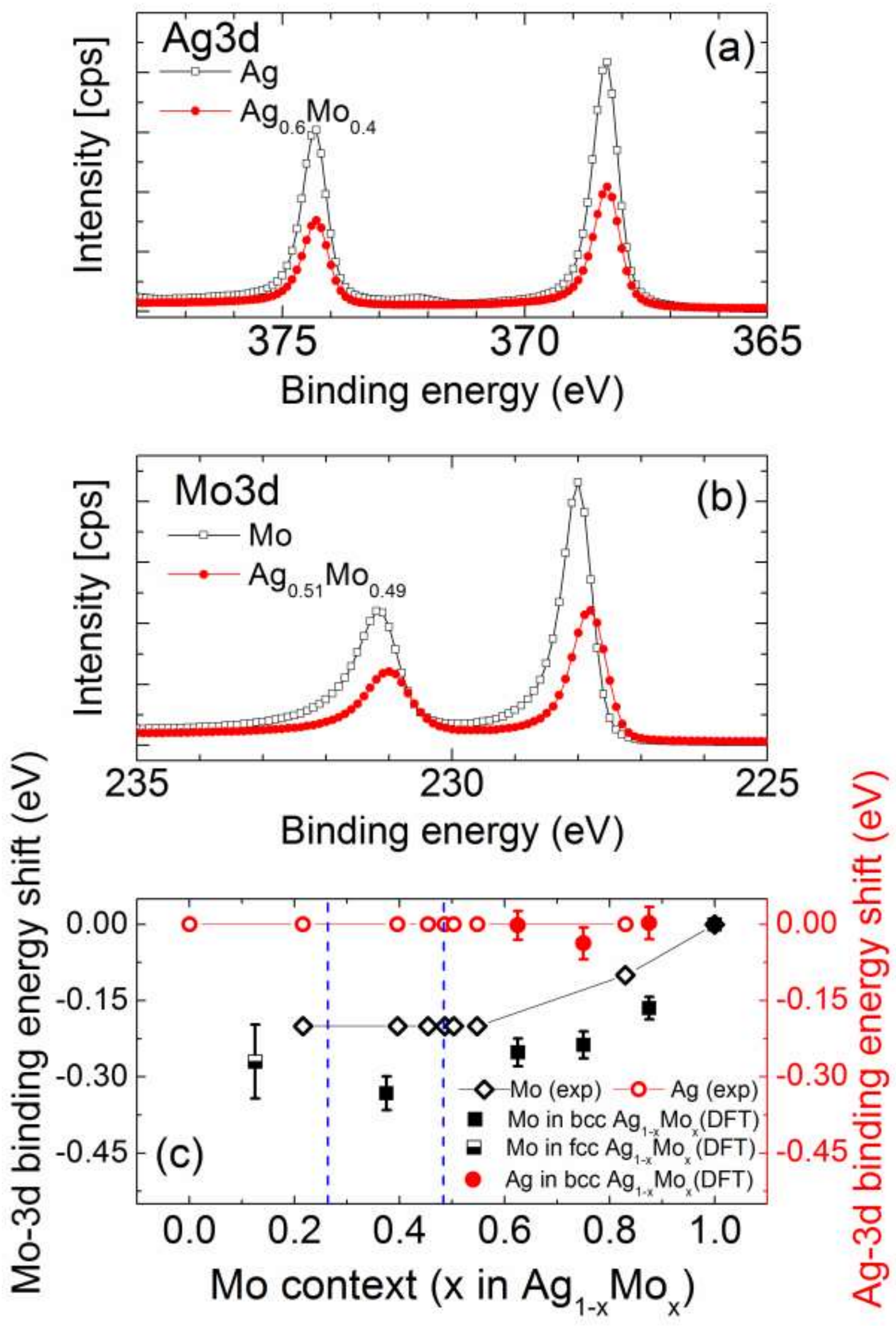
Fig. 4. (Color online) Characteristic (a) Ag 3d and (b) Mo 3d core level scans at various compositions. (c) Mo 3d and Ag 3d core level shifts obtained from XPS (exp) along with DFT calculated shifts for the two crystal structures (fcc- and bcc-Ag 1 -xMox) as a function of Mo content $x$. The vertical dotted lines define the compositional range (as determined by XRD data) in which fcc- and bcc-Ag1-xMox metastable structures coexist. The error bars in the DFT data correspond to a $95 \%$ confidence interval for the mean position of the core-level shift. The error of the experimental XPS data was estimated to be $\pm 0.025 \mathrm{eV}$. 